CARNETS OE Carnets de géographes

GÉOGRAPHES.

\title{
Géographie ludique de fa France
}

Approche spatiale des pratiquants et des fédérations de jeux institutionnels

\section{Manouk Borzakian}

\section{QpenEdition}

\section{Journals}

Édition électronique

URL : http://journals.openedition.org/cdg/2847

DOI : $10.4000 /$ cdg. 2847

ISSN : 2107-7266

Éditeur

UMR 245 - CESSMA

Référence électronique

Manouk Borzakian, « Géographie ludique de fa France », Carnets de géographes [En ligne], 2 | 2011, mis en ligne le 02 mars 2011, consulté le 07 mai 2019. URL : http://journals.openedition.org/cdg/2847 DOI : $10.4000 /$ cdg. 2847

Ce document a été généré automatiquement le 7 mai 2019.

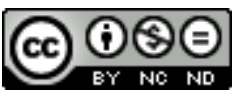

La revue Carnets de géographes est mise à disposition selon les termes de la Licence Creative Commons Attribution - Pas d'Utilisation Commerciale - Pas de Modification 4.0 International. 


\title{
Géographie ludique de fa France
}

\author{
Approche spatiale des pratiquants et des fédérations de jeux \\ institutionnels
}

Manouk Borzakian

1 Dans la lignée des travaux de géographie du sport en France (AUGUSTIN 1995) et dans les pays anglo-saxons (BALE 2003), est née l'idée d'une géographie des pratiques ludiques en France, suivant un postulat défendu par plusieurs grandes figures des sciences sociales (CAILLOIS 1958, GEERTZ 1973) : les jeux nous informent sur les cultures qui les inventent, les adoptent ou les rejettent. Postulat qui permet d'inclure ce travail sur les pratiques ludiques en France dans deux questionnements plus larges, l'un sur l'articulation du local et du global, autrement dit la supposée uniformisation culturelle liée à la mondialisation (WARNIER 2007), l'autre sur les modalités d'adoption de telle ou telle pratique ou innovation par certains groupes, soit la rencontre entre une offre et une demande culturelles (POCIELLO 1981).

D'où la problématique suivante : peut-on identifier en France des « régions ludiques », ou plus largement une hétérogénéité spatiale des pratiques ludiques? S'ajoute une question corollaire: quels sont les facteurs permettant d'expliquer ces contrastes? Cette problématique repose sur l'hypothèse que les espaces différenciés des pratiques ludiques sont produits conjointement par des déterminants exogènes de type historique, sociologique, etc., par des éléments spécifiquement spatiaux (AUGUSTIN ET ALII 2008) et, enfin, par des caractéristiques propres aux jeux eux-mêmes, leur "logique interne" (PARLEBAS 1999, 2003).

3 Pour poser les bases de cette recherche, un premier détour consiste en une redéfinition des jeux - et, en leur sein, des jeux institutionnels, régis par des fédérations nationales et internationales. Cela implique une relecture critique des deux ouvrages fondateurs (HUIZINGA 1938, CAILLOIS 1958) et de leurs nombreux commentaires, qui n'ont que rarement su signaler les impasses liées à un projet mal formulé et à certains présupposés, imputables en particulier aux biais du vocabulaire traitant des jeux (CALVET 1978) : il faut distinguer le jeu, concept large et incertain, et les jeux, objets clairement identifiables sur la pratique desquels peut s'interroger le chercheur. 
Un deuxième détour implique d'établir comment se fait le lien entre la logique interne des jeux - leurs règles et mécanismes - et les cultures qui les accueillent. Soit d'identifier des pistes pour une géographie des pratiques ludiques, dont les facteurs explicatifs seraient à trouver, entre autres, au sein des jeux eux-mêmes, notamment du fait des représentations spatiales qu'ils traduisent, mais également des valeurs qui leur sont attachées.

5 Sur cette base, est possible une enquête de terrain portant sur six fédérations (bridge, dames, échecs, go, scrabble et tarot). Elle est tout d'abord inévitablement quantitative, du fait de l'échelle retenue, et aboutit en premier lieu à un travail de description des logiques spatiales des pratiques ludiques. À l'échelle du monde, on retrouve, à propos des pratiques ludiques, les ambiguïtés de la mondialisation culturelle, entre, d'une part, l'imposition d'un modèle occidental (via le jeu d'échecs) et, d'autre part, la résistance de pratiques locales (par exemple le tarot en France), le succès en Occident de jeux importés (le go), ou encore la réappropriation de certains jeux par les populations des pays d'accueil.

6 Il n'est pas plus question d'uniformisation des pratiques ludiques à l'échelle française que mondiale : se dessinent des espaces de pratique fortement hétérogènes, avec des régions de forte concentration - Alsace, Île-de-France et Côte d'Azur pour les échecs, Ouest parisien et Côte d'Azur pour le bridge - et, inversement, des espaces où certaines pratiques sont nettement sous-représentées. Logiquement, ces contrastes sont d'autant plus marqués pour les jeux moins pratiqués, en particulier les dames, repliées sur quelques bastions du Nord de la France, le go, concentré autour des métropoles françaises, et enfin le tarot, très représenté en Bourgogne et Franche-Comté.

7 Ensuite, une investigation à la fois quantitative (sur des données socio-économiques, en particulier) et qualitative (par voie d'entretiens semi-directifs), a permis d'identifier des facteurs explicatifs. Premièrement, l'étude de la relation entre démographie et pratiques ludiques permet de distinguer trois catégories: bridge et go entretiennent un lien structurel avec l'urbanisation, surtout le second, dont l'introduction en France est récente et la diffusion hiérarchique en cours. À l'inverse, la pratique institutionnalisée du scrabble et surtout du tarot est favorisée dans les espaces ruraux et périurbains, ainsi que les petites villes - où l'on peut opposer une pratique masculine du tarot et féminine du scrabble. Enfin, échecs et dames constituent un type intermédiaire, peu concerné par la démographie.

8 S'ajoutent des déterminants d'ordre social et culturel. On observe, en reprenant les apports de la sociologie structuraliste (BOURDIEU 1979), que le bridge s'affirme comme un jeu prisé par les franges les plus favorisées de la société : sa géographie est celle des quartiers riches des grandes métropoles, des stations balnéaires et des villes thermales. De même, le go apparaît comme un jeu confidentiel, en partie réservé à une élite intellectuelle soucieuse de se distinguer par cette pratique empreinte d'exotisme. Inversement, les dames se caractérisent par une forte relégation sociale, qui participe à expliquer leur géographie. Une conclusion similaire, quoique plus nuancée, vaut pour le scrabble et le tarot.

9 Cette lecture sociologique des pratiques ludiques, de même qu'une approche culturaliste - qui participe à expliquer le succès des échecs dans l'Alsace protestante et bourgeoise souffrent toutefois de nombreuses exceptions et anomalies, liées le plus souvent à l'impact sur les moyen et long termes d'initiatives individuelles, tendant à affirmer le 
poids des acteurs et de la conjoncture face aux déterminants sociaux et culturels: l'explosion de la pratique des échecs en Corse, depuis une génération, va clairement dans ce sens.

C'est là que se situe la conclusion la plus digne d'intérêt de ce travail, qui montre que la géographie des faits de culture ne peut s'envisager que dans une perspective qui tienne compte à la fois d'éléments structurels - lois de l'espace, déterminismes sociaux et culturels - et de la capacité des acteurs à dépasser et remettre en cause ces derniers, le tout sans négliger le rôle, à différentes échelles, du hasard, au sens d'une synchronisation imprévisible entre des chaînes de causalité indépendantes (BOUDON 1984), comme moteur de la rencontre entre offre et demande culturelles.

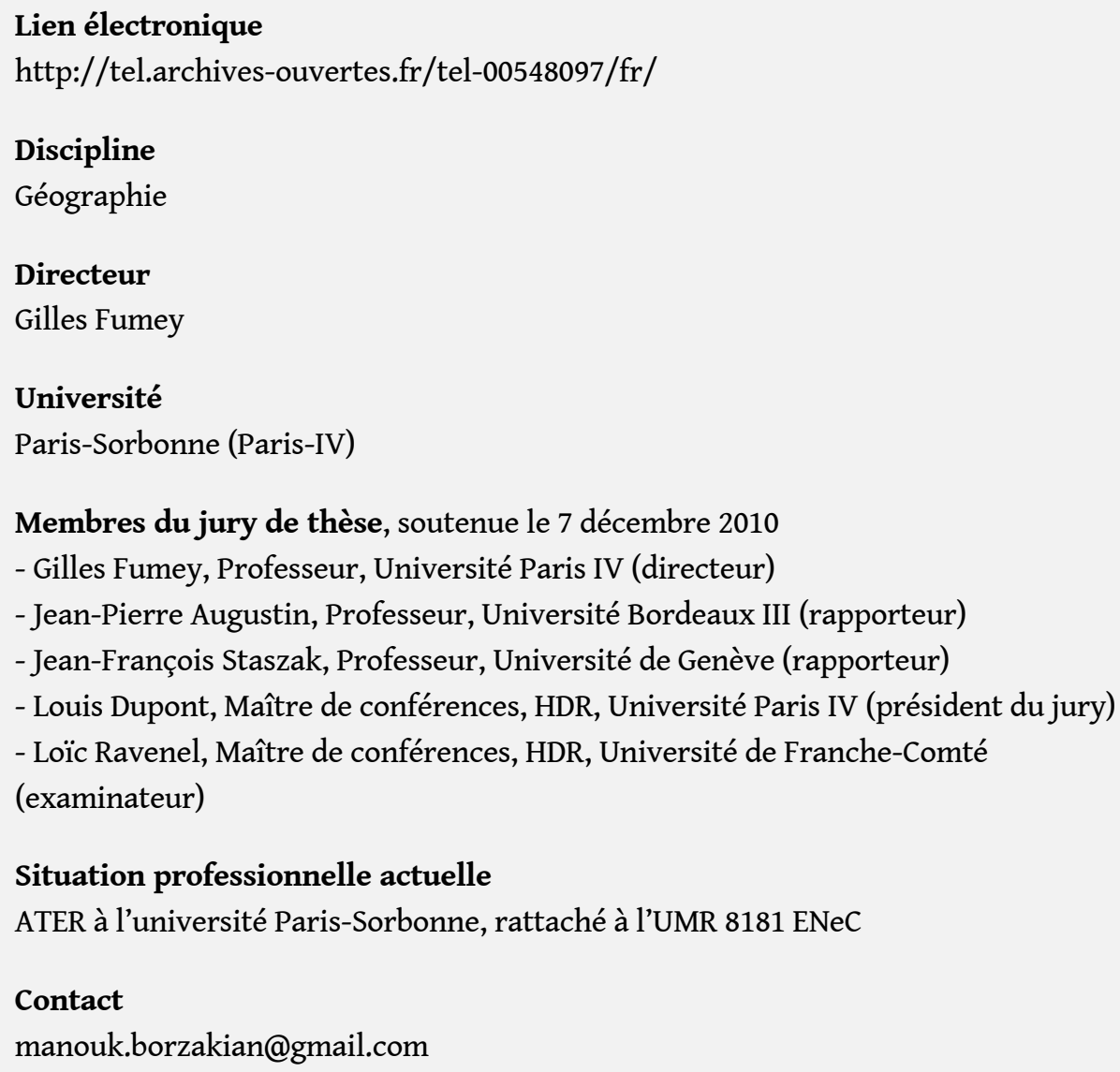

\section{BIBLIOGRAPHIE}

AUGUSTIN J.-P., 1995, Sport, géographie et aménagement, Paris, Nathan, 254 pp.

AUGUSTIN J.-P., BOURDEAU P., RAVENEL L., 2008, Géographie des sports en France, Paris, Vuibert, $180 \mathrm{pp}$. 
BALE J., 2003, Sports Geography, London/New York, Routledge, 196 pp. BOUDON R., 1991 [1984], La Place du désordre, Paris, PUF, 246 pp.

BOURDIEU P., 1979, La Distinction. Critique sociale du jugement, Paris, Minuit, 670 pp. CAILLOIS R., 1967 [1958], Les Jeux et les hommes, Paris, Gallimard (2ème édition), 374 pp. CALVET L.-J., 1978, Les Jeux de la société, Paris, Payot, 226 pp.

GEERTZ C., 1983 [1973], « Jeu d'enfer. Notes sur le combat de coqs balinais », dans Bali, interprétation d'une culture, Paris, Gallimard, pp. 165-215.

HUIZINGA J., 1951 [1938], Homo Ludens. Essai sur la fonction sociale du jeu, Paris, Gallimard, 342 pp.

PARLEBAS P., 1999, Jeux, sports et société. Lexique de praxéologie motrice, Paris, INSEP, 460 pp.

PARLEBAS P., 2003, «Le destin des jeux : héritage et filiation », Socio-anthropologie, $\mathrm{n}^{\circ} 13$, [en ligne] http://socio-anthropologie.revues.org/index173.html.

POCIELLO C., 1981, « Nouvelles approches », dans Pociello C. (dir.), Sports et société. Approche socioculturelle des pratiques, Paris, Vigot, pp. 9-29.

WARNIER J.-P., 2007, La Mondialisation de la culture, Paris, La Découverte, 126 pp.

INDEX

Thèmes : Carnets de soutenances 